\title{
STUDY OF HUMORAL AND CELLULAR IMMUNE RESPONSE IN FMD VACCINATED SHEEP WITH DIFFERENT INACTIVATED FMD VACCINES
}

\author{
ABEER H.M. HASSAN ${ }^{1}$, Y.A. EL-SINOSY ${ }^{2}$, AFAF D. ABDEL MAGEIID ${ }^{2}$, \\ A.A. AGOOR ${ }^{1}$ and ABEER E. MAHMOUD ${ }^{1}$
}

1. Veterinary Serum and Vaccine Research Institute, Abbasia, Cairo

2. Biochemistry Department, Faculty of Veterinary Medicine, Benha University

(Manuscript received 27 February 2017)

\begin{abstract}
$\mathrm{T}$ his study has been designed as a trial for comparing of ISA206 adjuvanted Foot and Mouth Disease (FMD) vaccine (commercial vaccine), commercial FMD vaccine containing vitamin $(A)$, commercial FMD vaccine containing vitamin (ESelenium) and commercial FMD vaccine containing both vitamin (A) and vitamin (E-Selenium) together in eliciting immunity. The obtained results revealed that the duration of humoral immunity elicited by commercial vaccine only was 30 weeks, while, it was 32 weeks in commercial vaccine containing vitamin (A), 36 weeks in commercial vaccine containing vitamin (E-Selenium) and 38 weeks in commercial vaccine containing both vitamin (A) and vitamin (ESelenium) together in vaccinated sheep. Results of cellular immunity evaluation indicated that mean optical density (mean OD) correspond to non-specific mitogens using MTT kit reached its highest values by $21^{\text {st }}$ day post vaccination in all vaccinated sheep and mean OD values correspond to FMDV as specific mitogen were higher than that of non-specific mitogen. Also, the highest mean $O D$ values were obtained in vaccinated sheep with commercial vaccine containing both vitamin (A) and vitamin (E-Selenium) together. It could be concluded that usage of inactivated oil FMD vaccine using Montanide ISA 206 containing both vitamin (A) and vitamin (E-selenium) resulted in longer lasting immunity than that with Montanide ISA 206 only, besides the stimulating effect of both vitamin (A) and vitamin (E-selenium) in both humoral and cellular immunity.
\end{abstract}

\section{INTRODUCTION}

Foot and Mouth Disease (FMD) is a highly contagious disease and one of the most devastating diseases of cloven-hoofed animals, including domestic animals such as cattle, buffaloes, sheep, goats and pigs, as well as antelope, hison and other wild bovines and deer (OIE, 2012).

This disease has a significant economic impact on livestock industry worldwide. The majority of the economic losses results either from mortality of young animals, loss of milk and meat and drastic fall in production performance or indirect losses due to the imposition of trade restriction (Verma, 2008).

FMD virus is aphathovirus of Family Picornaviridae, There are seven recognized serotypes of FMD $(O, A, C$, Asia, SAT1, SAT2 and SAT3) which differ in 
distribution across the world (Pereira, 1977) and comprise more than 65 subtypes (Sharma and Kakkar, 2005).

In Egypt, the disease is enzootic and outbreaks have been reported since 1950. FMDV serotypes (SAT2, A) and (O) were last reported in the years 1950, 1972 and 2000, respectively (Aidaros, 2002). Type (O) was the most prevalent since 1960 and onwards (Farag et al., 2005).

Since 1950, 1953 and 1956 serotype (A) was not recorded in Egypt (Zahran, 1960). Recently, serotype (A) of FMD virus was introduced to Egypt through live animals importation and severe clinical signs occurred among cattle and buffaloes (Abd El-Rahman et al., 2006). Also, FMDV serotype (SAT2) outbreaks in Egypt were officially reported by OIE (2012). In addition to this, endemic serotypes $A$ and $O$ continue to circulate in country (Lockart et al., 2012 and Heba Attia, 2017).

Most of Foot and Mouth Disease vaccines are made of binary ethyleneimine (BEI) inactivated virus. Oil adjuvants are generally preferred due to introducing longer lasting immunity (Hunter, 1998). The commercial FMD vaccine is adjuvanted with Montanide ISA206 which is double water-in-oil-in-water (W/O/W) adjuvant eliciting protective humoral immune response and cellular immunity in vaccinated animals (Clo et al., 2008).

Kutukculer et al. (2000) reported that both vitamin A and vitamin E-selenium can be used as adjuvant inducing high serum neutralization antibody titers and stimulate cellular immunity. Also, Rajeesh et al. (2008) found that using vitamin Eselenium as an adjuvant elicits high and prolonged immune response in vaccinated animals.

This study is carried out as an attempt to compare between (ISA206), (ISA206 and vitamin A), (ISA206 and vitamin E-Selenium) and (ISA206 and both vitamin $A$ and vitamin $E$-Selenium together) vaccine in eliciting both humoral and cellular immunity.

\section{MATERIAL AND METHODS}

\section{Animals:}

Twenty five sheep of 6 months (local breed) were clinically healthy and free from antibodies against FMD as proved by using SNT and ELISA.

\section{FMD virus strains:}

Vaccinal strains serotypes O Pan Asia 2012, A/Iran 05, and SAT2/Egy/2012 of FMDV were used for production of trivalent FMD vaccines and neutralization test.

\section{FMD vaccines:}

\section{a. Commercial FMD vaccine:}

Inactivated FMD oil trivalent vaccine. 


\section{b. Commercial FMD vaccine containing vitamin (A):}

Inactivated FMD oil trivalent vaccine containing vitamin (A) 200,000 IU/dose (Semba et al., 1998).

\section{c. Commercial FMD vaccine containing vitamin (E-selenium):}

Inactivated FMD oil trivalent vaccine containing vitamin (E-Selenium) $500 \mathrm{mg} /$ dose (Milad et al., 2001).

d. Commercial FMD vaccine containing both vitamin (A) and vitamin (Eselenium:

Inactivated FMD oil trivalent vaccine containing both vitamin (A) 200,000IU/dose and vitamin (E- selenium) $500 \mathrm{mg} /$ dose.

\section{Experimental Design:}

Four groups of sheep (5 animals/group) were vaccinated with the tested vaccines. Five animals were kept without vaccination as a control negative group. Serum samples were collected weekly post vaccination for one month and then every 2 weeks until the end of the experiment (38 weeks). Heparinized blood samples for MTT assay of cell mediated immunity were also collected.

\section{Estimation of the immune response:}

\subsection{Humoral immunity:}

It was estimated using SNT according to OIE Manual (2012) and ELISA according to Hamblin et al. (1986).

\subsection{Cellular immunity:}

It was estimated using MTT kit according to Verma (2010).

\section{Statistical analyses}

Results were analyzed using ANOVA test (Sendecor, 1971).

\section{RESULTS}

From Tables (1 to 6 ), the results of humoral immune response revealed that mean protective serum antibody titre evaluated using SNT and ELISA were as follows:

The first group reached the SNT protective level $\left(1.5 \log _{10}\right)$ at $3^{\text {rd }}$ week post vaccination (WPV) with mean antibody titers of (1.68 $\log _{10} / \mathrm{ml}$ for "O" serotype, 1.68 $\log _{10} / \mathrm{ml}$ for "A" serotype) and at the $4^{\text {th }}$ WPV $\left(1.59 \log _{10} / \mathrm{ml}\right)$ in case of "SAT2" serotype and to ELISA protective level $\left(1.65 \log _{10}\right)$ at the $3^{\text {rd }}$ WPV $\left(1.89 \log _{10} / \mathrm{ml}\right.$ for "O" serotype and $1.68 \log _{10} / \mathrm{ml}$ for "SAT2" serotype) and $1.59 \log _{10} / \mathrm{ml}$ for "A" serotype at the $2^{\text {nd }}$ WPV.

The highest level of mean antibody titers were obtained at $10^{\text {th }}$ week post vaccination (WPV) (2.64 $\log _{10} / \mathrm{ml}$ for "O" serotype, $2.67 \log _{10} / \mathrm{ml}$ for "A" serotype and $2.46 \log _{10} / \mathrm{ml}$ for "SAT2" serotype) by SNT and by ELISA (2.73 $\log _{10} / \mathrm{ml}$ for "O" serotype, $2.67 \log _{10} / \mathrm{ml}$ for "A" serotype and $2.46 \log _{10} / \mathrm{ml}$ for "SAT2" serotype). 
The duration of protection lasted for 30, 36 and 28 WPV for "O", "A" and "SAT2" serotypes respectively in SNT and for 28, 32 and 26 WPV in ELISA respectively.

In the second group, FMD SNT antibody protective levels were obtained at the $3^{\text {rd }}, 2^{\text {nd }}$ and $4^{\text {th }}$ WPV in "O", "A" and "SAT2" serotypes respectively while in case of ELISA they achieved at the $3^{\text {rd }}$ WPV in "O" and "SAT2" serotypes, but at the $2^{\text {nd }}$ WPV in "A" serotype. The highest level was recorded at $10^{\text {th }}$ WPV $(2.67,2.73$ and 2.43 $\log _{10} / \mathrm{ml}$ for "O", "A" and "SAT2" serotypes respectively) by SNT and (2.76 and 2.73 $\log _{10} / \mathrm{ml}$ for "O" and "A" serotypes respectively) and at the $12^{\text {th }}$ WPV $\left(2.55 \log _{10} / \mathrm{ml}\right.$ for "SAT2" serotype) by ELISA with protection duration for 32, 36 and 30 WPV for "O", "A" and "SAT2" serotypes respectively in SNT and for 30, 36 and 28 WPV in ELISA respectively.

Regarding to the third group, SNT antibody protective levels were recorded at the $3^{\text {rd }}, 2^{\text {nd }}$ and $3^{\text {rd }}$ WPV in "O", "A" and "SAT2" serotypes respectively while in case of ELISA they achieved at the $2^{\text {nd }}$ WPV in "O" and "A" serotypes, but at the $3^{\text {rd }}$ WPV in "SAT2" serotype. The highest level of mean SNT antibody titers were recorded at the $10^{\text {th }}$ WPV (2.85 $\log _{10} / \mathrm{ml}$ for "O" serotype, $2.94 \log _{10} / \mathrm{ml}$ for "A" serotype and 2.55 $\log _{10} / \mathrm{ml}$ for "SAT2" serotype) by SNT and $\left(2.94 \log _{10} / \mathrm{ml}\right.$ for "O" serotype and 2.55 for "SAT2" serotype) and at the $12^{\text {th }}$ WPV (3.00 $\log _{10} / \mathrm{ml}$ for "A" serotype) by ELISA with protection duration lasted for 36, 38 and 32 WPV for "O", "A" and "SAT2" serotypes respectively in SNT and for 32, 36 and 30 WPV in ELISA respectively.

In the fourth group, the SNT protective levels reached at $3^{\text {rd }}$ WPV with mean antibody titers of $1.77 \log _{10} / \mathrm{ml}$ for "O" serotype and $1.53 \log _{10} / \mathrm{ml}$ for "SAT2" serotype $)$ and at the $2^{\text {nd }}$ WPV $\left(1.71 \log _{10} / \mathrm{ml}\right)$ in case of "A" serotype and to ELISA protective level at the $2^{\text {nd }}, 1^{\text {st }}$ and $3^{\text {rd }}$ WPV $\left(1.68,1.68\right.$ and $1.74 \log _{10} / \mathrm{ml}$ for "O", "A" and "SAT2" serotypes respectively).

The highest level of mean SNT antibody titers were gained at the $10^{\text {th }}$ to $12^{\text {th }}$ WPV (2.94 $\log _{10} / \mathrm{ml}$ for "O" serotype, $2.94 \log _{10} / \mathrm{ml}$ for "A" serotype and $2.61 \log _{10} / \mathrm{ml}$ for "SAT2" serotype) by SNT and $\left(2.94 \log _{10} / \mathrm{ml}\right.$ for "O" serotype, $2.97 \log _{10} / \mathrm{ml}$ for "A" serotype and $2.64 \log _{10} / \mathrm{ml}$ for "SAT2" serotype) by ELISA with protection duration lasted for 38, 38 and 32 WPV for "O", "A" and "SAT2" serotypes respectively in SNT and for 38,38 and 26 WPV in ELISA respectively.

\section{DISCUSSION}

From Tables (1 to 6 ), the results revealed that SNT and ELISA titers of FMD antibodies induced by oil Montanide ISA-206 trivalent FMD vaccines in group one went in hand with Barteling and Vreeswij, 1991 and Sonia, 2007) who reported that oil emulsion FMD vaccine (double oil emulsion) induced best results in comparison with alhydragel vaccine. Also agree with Patil et al. (2002) and Abeer et al. (2009) who 
found that FMD vaccines adjuvanted with Montanide ISA-206 can promote long lasting immunity.

The obtained results in the $2^{\text {nd }}$ group in which animals were vaccinated with oil Montanide ISA-206 trivalent FMD vaccine containing vitamin (A), the results agree with Semba et al. (1992) who reported that administration of high dose of vitamin (A) resulted in significantly higher titers of anti-tetanus toxoid after immunization compared with those in control group.

In addition, in the trial of Rahman et al. (1999), they reported that vitamin (A) had a positive effect on the antibody response to diphtheria toxoid antigen in diphtheria vaccine. Also, Semba (1999) and Bahl et al. (2002) resulted that when vitamin (A) administration with Polivirus type 1 vaccine, the protective titer against type 1 poliovirus was significantly higher in experimental group. Semba et al. (2005) reported that vitamin $(A)$ has a great role in improving immunofunction by increasing antibody titer against Schwars Measles vaccine.

In the third group, in which animals were vaccinated with oil Montanide ISA206 trivalent FMD vaccine containing vitamin (E-selenium), the results are supported by Martin Nemec et al. (1990) and Cherly (1996) who found that vitamin (E-selenium) injection increased Ig production and stimulated cellular immunity. Weber et al. (2008) suggested that antibody formation against Newcastle disease was improved by vitamin E-selenium administration.

Also, Kanchana and Jeyanthi (2010) reported that when useding vitamin (Eselenium) as adjuvant for BVD vaccine, it provided higher level of protection.

In the fourth group, in which animals were vaccinated with oil Montanide ISA206 trivalent FMD vaccine containing both vitamin (A) and vitamin (E-selenium) together, the obtained results agreed with Kutukculer et al. (2000) who made Turkish trials for using vitamin (A), vitamin (E-selenium) and both vitamins (A) and (Eselenium) together as adjuvant and found that highest antibody response when used both vitamins (A) and (E-selenium) together as adjuvant.

The obtained results of cell mediated immune response using MTT kit for all animal groups expressed as mean Optical Density (mean OD) in table (7) showed that:

In the first group, mean ODs were $(0.121-0.231)$ by using non-specific mitogen and FMD viruses (as specific mitogen) at the $3^{\text {rd }}$ day post vaccination (DPV) and reached its highest level $(0.510-0.600)$ at $21^{\text {st }}$ day post vaccination for nonspecific mitogen and FMD viruses as (specific mitogen) respectively.

The results of first group were supported by those of Soos and Tuboly (1983) and Sharma et al. (1985) who reported that cell mediated immune response was a constituent of immune response against FMD virus and were in agreement in some points with Abeer et al. (2009) who stated that FMD vaccine stimulated the cellular 
immune response and lymphocyte stimulation by FMD as specific mitogen was greater than that by non-specific mitogen.

In the $2^{\text {nd }}$ group, the mean OD was $(0.197-0.289)$ at the $3^{\text {rd }}$ DPV and $(0.600-$ 0.710 ) at $21^{\text {st }}$ DPV for non-specific mitogen and FMDV as specific mitogen respectively.

These previously mentioned results were supported by Jason et al. (2002) who reported that vitamin "A" stimulate cellular response via stimulate T-cell proliferation.

Coutsoudis et al. (1992) reported that the beneficial effect of vitamin (A) supplementation with severe measles enhancing specific IgG antibody level and total lymphocyte proliferation. Stephensen (2001) reported that vitamin "A" stimulate the cell mediated activity by stimulating T-helper cell. Also, Ribeiro et al. (2003) found that vitamin "A" increase T-cell and B-cell count. Villamor and Fawzi (2005) found that vitamin "A" stimulate lymphocyte activation for cytokines production.

While, in the third group, mean OD was $(0.245-0.345)$ at the $3^{\text {rd }}$ DPV reached its highest value at $21^{\text {st }} \mathrm{DPV}(0.711-0.803)$ by using non-specific mitogen and FMDV (as specific mitogen) respectively.

These results agreed with Hogan et al. (1990) who reported that the Eselenium plays an important role in phagocytosis.

Howard et al. (1989) explained the mechanism of E-slenium by which enhances cellular immunity as it inhibits the generation of suppressor cell activity. Reffett Stabel et al. (1989) found that E-selenium administration increased Tlymphocyte blastogenesis in-vitro stimulation with mitogen in some studies. Also, Wuryastuti et al. (1993) reported that E-selenium increased the phagocytic activity and mitogenic response.

But in the $4^{\text {th }}$ group, mean OD was $(0.271-0.400)$ at $3^{\text {rd }}$ DPV and $(0.820-910)$ at $21^{\text {st }}$ DPV by using non-specific mitogen and FMD (as specific mitogen) respectively.

The last mentioned results come in parallel with those found by Tengerdy (1990) who used both vitamin (A) and (E-selenium) together as adjuvant in vaccine preparation resulting in maximizing of both the cellular and humoral immune response than dietary supplementation.

Also, Semba et al. (2005) and Kanchana and Jeyanthi (2010) reported that both vitamin (A) and vitamin (E-selenium) stimulate virus function of T-lymphocyte enhancing the immune response of the host and affecting macrophage activity and provided higher level of protection against natural challenges.

Finally, it could be concluded that administration of inactivated oil vaccine with Montanide ISA 206, both vitamin (A) and vitamin (E-selenium) together resulted in longer lasting immunity than that with Montanide ISA 206 only, in addition to the stimulating effect of both vitamin (A) and vitamin (E-selenium) in both humoral and cellular immunity. 
Table 1. Mean serum neutralization antibody titer against FMD serotype (O) in different sheep groups

\begin{tabular}{|c|c|c|c|c|c|}
\hline \multirow{2}{*}{$\begin{array}{l}\text { Weeks Post } \\
\text { Vaccination }\end{array}$} & \multicolumn{5}{|c|}{ Sheep Groups } \\
\cline { 2 - 6 } & Group (1) & Group (2) & Group (3)* & Group (4)** & $\begin{array}{c}\text { Control -ve } \\
\text { sheep group }\end{array}$ \\
\hline 0 & 0.17 & 0.15 & 0.14 & 0.14 & 0.15 \\
\hline 1 & 0.87 & 0.87 & 1.10 & 0.96 & 0.20 \\
\hline 2 & 1.29 & 1.35 & 1.41 & 1.44 & 0.10 \\
\hline 3 & 1.68 & 1.71 & 1.74 & 1.77 & 0.15 \\
\hline 4 & 1.89 & 2.01 & 2.04 & 2.10 & 0.25 \\
\hline 6 & 2.40 & 2.43 & 2.46 & 2.49 & 0.20 \\
\hline 8 & 2.46 & 2.61 & 2.67 & 2.70 & 0.15 \\
\hline 10 & 2.64 & 2.67 & 2.85 & 2.94 & 0.10 \\
\hline 12 & 2.52 & 2.52 & 2.79 & 2.91 & 0.15 \\
\hline 14 & 2.37 & 2.43 & 2.52 & 2.79 & 0.20 \\
\hline 16 & 2.22 & 2.31 & 2.49 & 2.67 & 0.30 \\
\hline 18 & 2.04 & 2.19 & 2.49 & 2.64 & 0.15 \\
\hline 20 & 2.07 & 2.10 & 2.34 & 2.46 & 0.20 \\
\hline 22 & 1.89 & 2.01 & 2.19 & 2.34 & 0.20 \\
\hline 24 & 1.81 & 1.89 & 2.10 & 2.22 & 0.25 \\
\hline 26 & 1.74 & 1.80 & 2.01 & 2.07 & 0.10 \\
\hline 28 & 1.68 & 1.74 & 1.86 & 1.92 & 0.10 \\
\hline 30 & 1.59 & 1.68 & 1.74 & 1.80 & 0.15 \\
\hline 32 & 1.47 & 1.59 & 1.65 & 1.74 & 0.10 \\
\hline 34 & 1.38 & 1.47 & 1.56 & 1.65 & 0.15 \\
\hline 36 & 1.29 & 1.41 & 1.53 & 1.59 & 0.10 \\
\hline 38 & 1.17 & 1.29 & 1.41 & 1.53 & 0.10 \\
\hline
\end{tabular}

Group (1): Sheep vaccinated with inactivated oil trivalent FMD vaccine, (Control +ve)

Group (2): Sheep vaccinated with inactivated oil trivalent FMD vaccine containing (Vit. A)

Group (3): Sheep vaccinated with inactivated oil trivalent FMD vaccine containing (Vit. E-selenium)

Group (4): Sheep vaccinated with inactivated oil trivalent FMD vaccine containing (Vit. A \& E-selenium)

- Serum neutralization antibody titer was expressed as $\log _{10}$

- $\quad$ Protective serum antibody titer $=1.5 \log _{10}$ according to OIE (2012)

* There is a significant increase between Group (4) and Group (3), also between Group (3) and Group (2) (at $\mathrm{P}<0.05$ )

** There is a significant increase between Group (4) and Group (1), also between Group (4) and Group (2) (at $P<0.01$ )

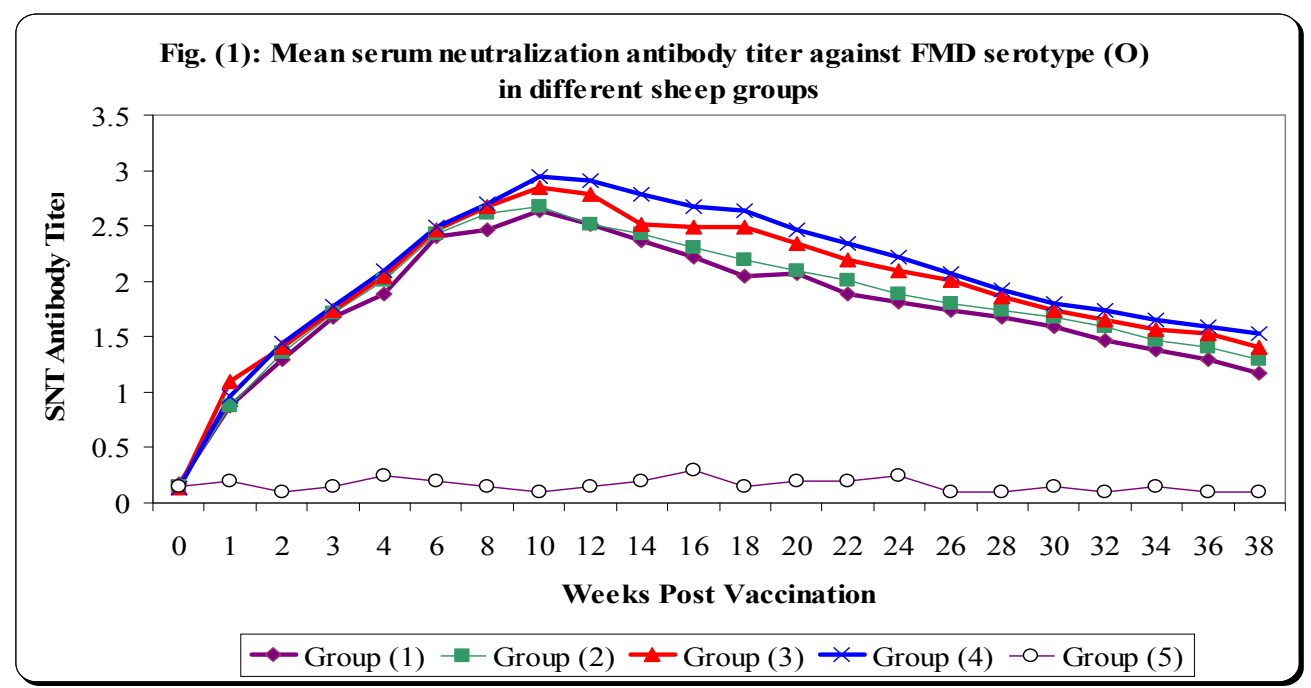


Table 2. Mean ELISA antibody titer against FMD serotype $(0)$ in different sheep groups

\begin{tabular}{|c|c|c|c|c|c|}
\hline \multirow{2}{*}{$\begin{array}{l}\text { Veeks Post } \\
\text { Vaccination }\end{array}$} & \multicolumn{5}{|c|}{ Sheep Groups } \\
\cline { 2 - 6 } & Group (1) & Group (2) & Group (3)* & Group (4)** & $\begin{array}{c}\text { Control -ve } \\
\text { sheep group }\end{array}$ \\
\hline 0 & 0.17 & 0.23 & 0.42 & 0.15 & 0.15 \\
\hline 1 & 1.17 & 1.17 & 1.26 & 1.38 & 0.25 \\
\hline 2 & 1.53 & 1.59 & 1.71 & 1.68 & 0.25 \\
\hline 3 & 1.89 & 1.98 & 2.04 & 2.07 & 0.20 \\
\hline 4 & 2.13 & 2.19 & 2.19 & 2.31 & 0.25 \\
\hline 6 & 2.34 & 2.40 & 2.25 & 2.49 & 0.30 \\
\hline 8 & 2.49 & 2.70 & 2.76 & 2.79 & 0.25 \\
\hline 10 & 2.73 & 2.76 & 2.94 & 2.94 & 0.15 \\
\hline 12 & 2.58 & 2.64 & 2.91 & 2.94 & 0.25 \\
\hline 14 & 2.34 & 2.43 & 2.76 & 2.82 & 0.25 \\
\hline 16 & 2.25 & 2.40 & 2.61 & 2.70 & 0.30 \\
\hline 18 & 2.04 & 2.22 & 2.52 & 2.67 & 0.30 \\
\hline 20 & 1.98 & 2.16 & 2.40 & 2.49 & 0.20 \\
\hline 22 & 1.80 & 2.10 & 2.25 & 2.37 & 0.25 \\
\hline 24 & 1.80 & 1.98 & 2.16 & 2.25 & 0.20 \\
\hline 26 & 1.65 & 1.86 & 2.10 & 2.19 & 0.20 \\
\hline 28 & 1.65 & 1.80 & 1.92 & 2.11 & 0.20 \\
\hline 30 & 1.47 & 1.68 & 1.86 & 2.07 & 0.25 \\
\hline 32 & 1.41 & 1.56 & 1.77 & 1.98 & 0.25 \\
\hline 34 & 1.26 & 1.50 & 1.62 & 1.92 & 0.20 \\
\hline 36 & 1.26 & 1.44 & 1.56 & 1.83 & 0.20 \\
\hline 38 & 1.05 & 1.32 & 1.50 & 1.71 & 0.20 \\
\hline
\end{tabular}

Group (1): Sheep vaccinated with inactivated oil trivalent FMD vaccine, (Control +ve)

Group (2): Sheep vaccinated with inactivated oil trivalent FMD vaccine containing (Vit. A)

Group (3): Sheep vaccinated with inactivated oil trivalent FMD vaccine containing (Vit. E-selenium)

Group (4): Sheep vaccinated with inactivated oil trivalent FMD vaccine containing (Vit. A \& E-selenium)

- ELISA antibody titer was expressed as $\log _{10}$

- $\quad$ Protective serum antibody titer $=1.65 \log _{10}$ according to OIE (2012)

* There is a significant increase between Group (4) and Group (3), also between Group (3) and Group (2) (at $\mathrm{P}<0.05$ )

** There is a significant increase between Group (4) and Group (1), also between Group (4) and Group (2) $($ at $\mathrm{P}<0.01)$

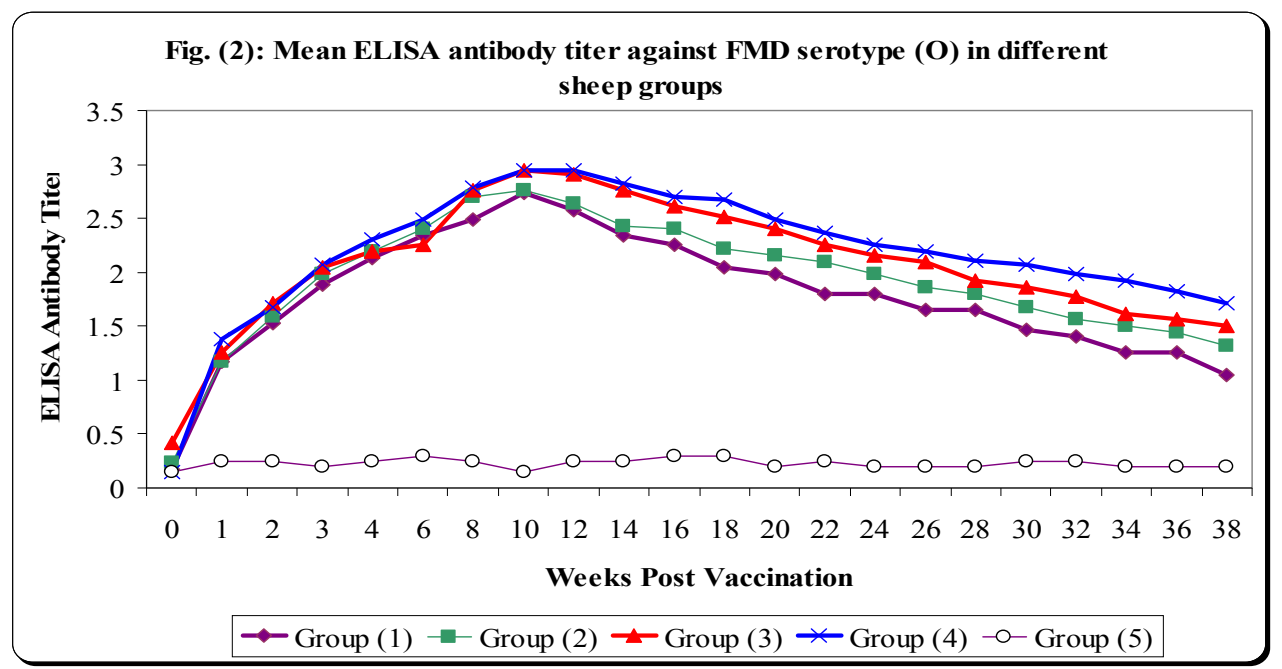


Table 3. Mean serum neutralization antibody titer against FMD serotype $(A)$ in different sheep groups

\begin{tabular}{|c|c|c|c|c|c|}
\hline \multirow{2}{*}{$\begin{array}{l}\text { Weeks Post } \\
\text { Vaccination }\end{array}$} & \multicolumn{5}{|c|}{ Sheep Groups } \\
\hline & Group (1) & Group (2) & Group (3)* & Group (4)** & $\begin{array}{l}\text { Control -ve } \\
\text { sheep group }\end{array}$ \\
\hline 0 & 0.20 & 0.14 & 0.14 & 0.23 & 0.30 \\
\hline 1 & 1.08 & 1.26 & 1.32 & 1.38 & 0.30 \\
\hline 2 & 1.38 & 1.53 & 1.62 & 1.71 & 0.15 \\
\hline 3 & 1.68 & 1.77 & 1.83 & 1.86 & 0.10 \\
\hline 4 & 1.92 & 2.01 & 2.10 & 2.13 & 0.10 \\
\hline 6 & 2.22 & 2.31 & 2.34 & 2.40 & 0.25 \\
\hline 8 & 2.52 & 2.58 & 2.64 & 2.67 & 0.30 \\
\hline 10 & 2.67 & 2.73 & 2.94 & 2.87 & 0.30 \\
\hline 12 & 2.64 & 2.67 & 2.94 & 2.94 & 0.10 \\
\hline 14 & 2.52 & 2.58 & 2.82 & 2.82 & 0.10 \\
\hline 16 & 2.43 & 2.46 & 2.70 & 2.76 & 0.30 \\
\hline 18 & 2.34 & 2.40 & 2.58 & 2.64 & 0.15 \\
\hline 20 & 2.16 & 2.16 & 2.46 & 2.55 & 0.15 \\
\hline 22 & 1.95 & 2.16 & 2.22 & 2.46 & 0.30 \\
\hline 24 & 1.98 & 2.07 & 2.19 & 2.43 & 0.30 \\
\hline 26 & 1.92 & 1.98 & 2.01 & 2.28 & 0.10 \\
\hline 28 & 1.83 & 1.86 & 1.92 & 2.04 & 0.10 \\
\hline 30 & 1.74 & 1.77 & 1.83 & 2.04 & 0.15 \\
\hline 32 & 1.65 & 1.71 & 1.71 & 1.89 & 0.25 \\
\hline 34 & 1.56 & 1.59 & 1.65 & 1.89 & 0.25 \\
\hline 36 & 1.50 & 1.53 & 1.56 & 1.74 & 0.10 \\
\hline 38 & 1.41 & 1.44 & 1.50 & 1.59 & 0.10 \\
\hline
\end{tabular}

Group (1): Sheep vaccinated with inactivated oil trivalent FMD vaccine, (Control +ve)

Group (2): Sheep vaccinated with inactivated oil trivalent FMD vaccine containing (Vit. A)

Group (3): Sheep vaccinated with inactivated oil trivalent FMD vaccine containing (Vit. E-selenium)

Group (4): Sheep vaccinated with inactivated oil trivalent FMD vaccine containing (Vit. A \& E-selenium)

- Serum neutralization antibody titer was expressed as $\log _{10}$

- $\quad$ Protective serum antibody titer $=1.5 \log _{10}$ according to OIE (2012)

* There is a significant increase between Group (4) and Group (3), also between Group (3) and Group (2) (at $\mathrm{P}<0.05$ )

** There is a significant increase between Group (4) and Group (1), also between Group (4) and Group (2) (at $\mathrm{P}<0.01$ )

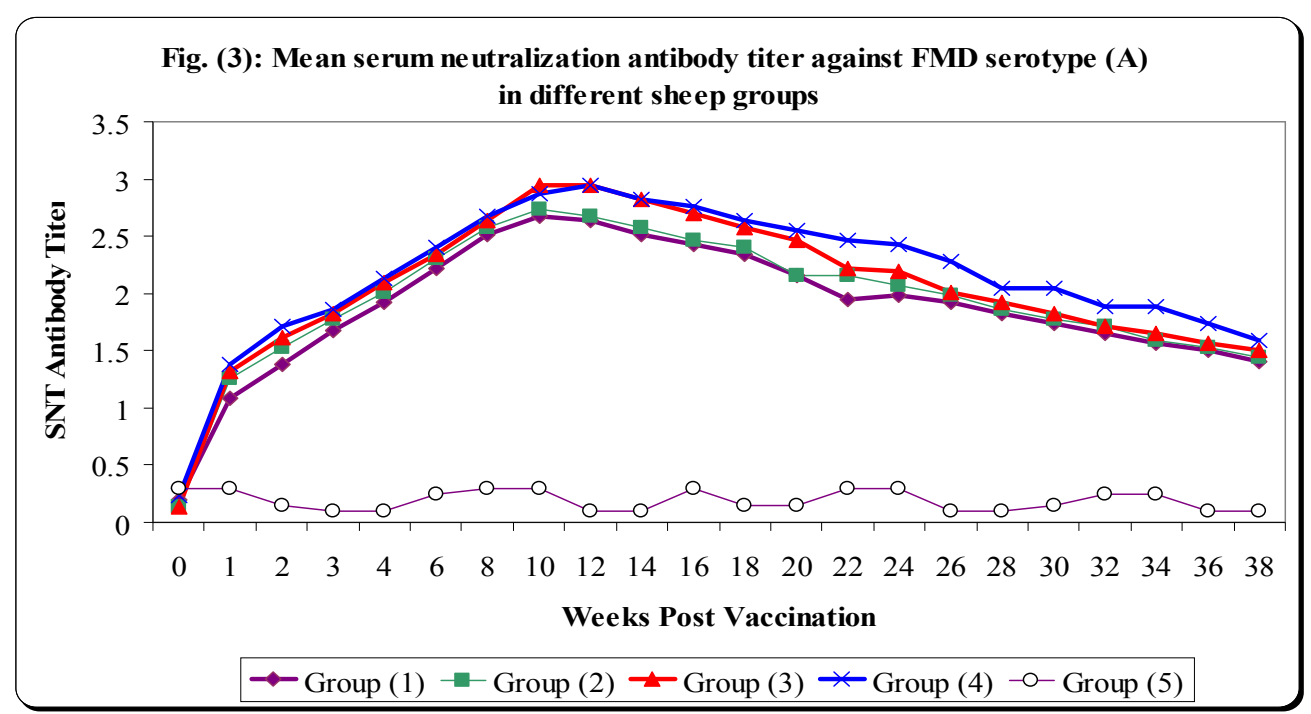


Table 4. Mean ELISA antibody titer against FMD serotype (A) in different sheep groups

\begin{tabular}{|c|c|c|c|c|c|}
\hline \multirow{2}{*}{$\begin{array}{l}\text { Weeks Post } \\
\text { Vaccination }\end{array}$} & \multicolumn{5}{|c|}{ Sheep Groups } \\
\cline { 2 - 6 } & Group (1) & Group (2) & Group (3)* & Group (4)** & $\begin{array}{c}\text { Control -ve } \\
\text { sheep group }\end{array}$ \\
\hline 0 & 0.19 & 0.20 & 0.20 & 0.19 & 0.15 \\
\hline 1 & 1.41 & 1.56 & 1.62 & 1.68 & 0.10 \\
\hline 2 & 1.59 & 1.83 & 1.92 & 2.01 & 0.30 \\
\hline 3 & 2.16 & 2.16 & 2.19 & 2.25 & 0.15 \\
\hline 4 & 2.37 & 2.34 & 2.43 & 2.46 & 0.30 \\
\hline 6 & 2.52 & 2.40 & 2.55 & 2.64 & 0.25 \\
\hline 8 & 2.67 & 2.73 & 2.82 & 2.82 & 0.30 \\
\hline 10 & 2.67 & 2.73 & 2.94 & 2.97 & 0.25 \\
\hline 12 & 2.40 & 2.70 & 3.00 & 2.97 & 0.20 \\
\hline 14 & 2.52 & 2.61 & 2.88 & 2.91 & 0.20 \\
\hline 16 & 2.46 & 2.52 & 2.73 & 2.79 & 0.25 \\
\hline 18 & 2.43 & 2.46 & 2.67 & 2.76 & 0.30 \\
\hline 20 & 2.34 & 2.28 & 2.52 & 2.64 & 0.25 \\
\hline 22 & 2.07 & 2.22 & 2.37 & 2.52 & 0.30 \\
\hline 24 & 2.07 & 2.16 & 2.34 & 2.40 & 0.15 \\
\hline 26 & 1.95 & 2.04 & 2.19 & 2.34 & 0.25 \\
\hline 28 & 1.92 & 1.98 & 2.07 & 2.19 & 0.30 \\
\hline 30 & 1.74 & 1.86 & 1.92 & 2.13 & 0.30 \\
\hline 32 & 1.71 & 1.80 & 1.89 & 2.01 & 0.30 \\
\hline 34 & 1.62 & 1.71 & 1.77 & 1.92 & 0.25 \\
\hline 36 & 1.62 & 1.68 & 1.74 & 1.83 & 0.20 \\
\hline 38 & 1.56 & 1.56 & 1.62 & 1.71 & 0.20 \\
\hline
\end{tabular}

Group (1): Sheep vaccinated with inactivated oil trivalent FMD vaccine, (Control +ve)

Group (2): Sheep vaccinated with inactivated oil trivalent FMD vaccine containing (Vit. A)

Group (3): Sheep vaccinated with inactivated oil trivalent FMD vaccine containing (Vit. E-selenium)

Group (4): Sheep vaccinated with inactivated oil trivalent FMD vaccine containing (Vit. A \& E-selenium)

- $\quad$ ELISA antibody titer was expressed as $\log _{10}$

- $\quad$ Protective serum antibody titer $=1.65 \log _{10}$ according to OIE (2012)

* There is a significant increase between Group (4) and Group (3), also between Group (3) and Group (2) (at $\mathrm{P}<0.05$ )

** There is a significant increase between Group (4) and Group (1), also between Group (4) and Group (2) (at $\mathrm{P}<0.01$ )

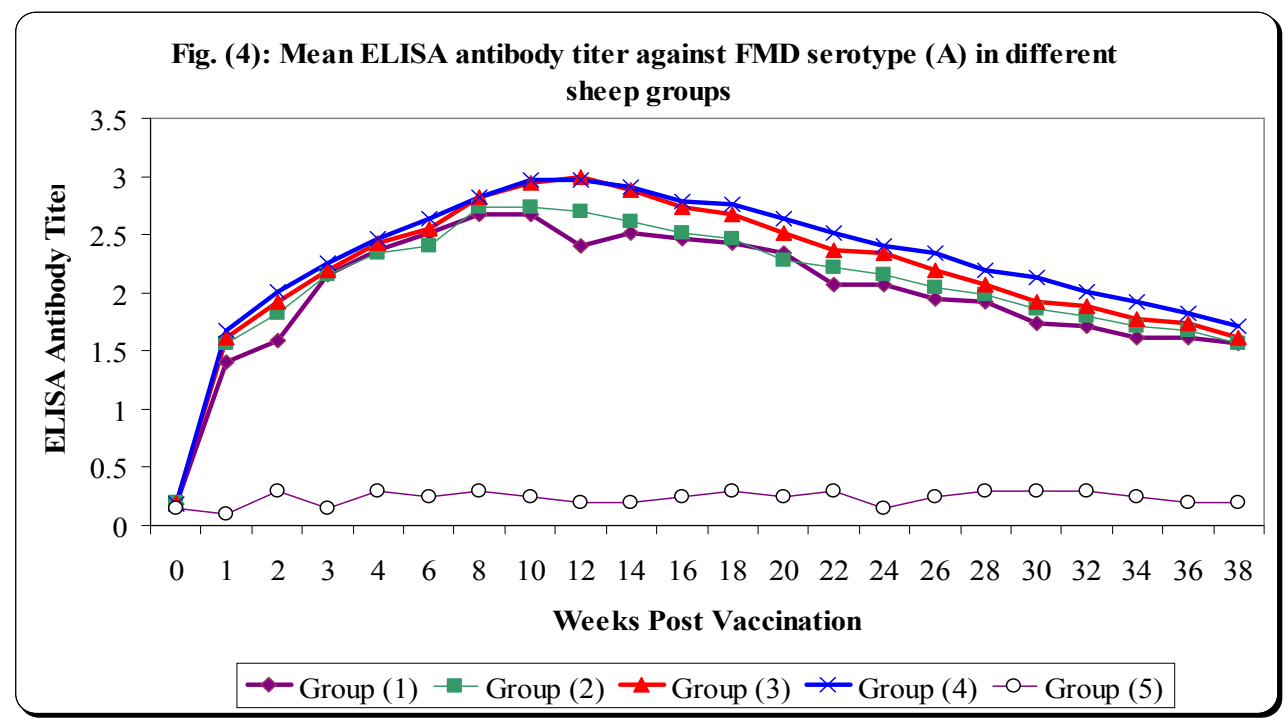


Table 5. Mean serum neutralization antibody titer against FMD serotype (SAT2) in different sheep groups

\begin{tabular}{|c|c|c|c|c|c|}
\hline \multirow[b]{2}{*}{$\begin{array}{l}\text { Weeks Post } \\
\text { Vaccination }\end{array}$} & \multicolumn{5}{|c|}{ Sheep Groups } \\
\hline & Group (1) & Group (2) & Group (3)* & Group (4)** & $\begin{array}{l}\text { Control -ve } \\
\text { sheep group }\end{array}$ \\
\hline 0 & 0.27 & 0.14 & 0.15 & 0.14 & 0.15 \\
\hline 1 & 0.66 & 0.66 & 0.78 & 0.78 & 0.10 \\
\hline 2 & 0.96 & 1.23 & 1.29 & 1.29 & 0.15 \\
\hline 3 & 1.26 & 1.41 & 1.56 & 1.53 & 0.10 \\
\hline 4 & 1.59 & 1.71 & 1.74 & 1.83 & 0.10 \\
\hline 6 & 1.86 & 1.95 & 2.01 & 2.07 & 0.25 \\
\hline 8 & 2.22 & 2.16 & 2.25 & 2.34 & 0.20 \\
\hline 10 & 2.46 & 2.43 & 2.55 & 2.61 & 0.15 \\
\hline 12 & 2.31 & 2.43 & 2.49 & 2.58 & 0.20 \\
\hline 14 & 2.25 & 2.28 & 2.34 & 2.46 & 0.15 \\
\hline 16 & 2.10 & 2.22 & 2.19 & 2.31 & 0.30 \\
\hline 18 & 2.04 & 2.07 & 2.07 & 2.16 & 0.20 \\
\hline 20 & 1.92 & 2.01 & 2.01 & 2.07 & 0.15 \\
\hline 22 & 1.80 & 1.86 & 1.89 & 1.92 & 0.10 \\
\hline 24 & 1.71 & 1.83 & 1.86 & 1.89 & 0.15 \\
\hline 26 & 1.56 & 1.68 & 1.71 & 1.74 & 0.20 \\
\hline 28 & 1.56 & 1.62 & 1.71 & 1.74 & 0.25 \\
\hline 30 & 1.41 & 1.53 & 1.56 & 1.59 & 0.15 \\
\hline 32 & 1.26 & 1.47 & 1.53 & 1.59 & 0.10 \\
\hline 34 & 1.26 & 1.32 & 1.41 & 1.44 & 0.15 \\
\hline 36 & 1.05 & 1.23 & 1.26 & 1.26 & 0.20 \\
\hline 38 & 0.96 & 1.14 & 1.20 & 1.26 & 0.20 \\
\hline
\end{tabular}

Group (1): Sheep vaccinated with inactivated oil trivalent FMD vaccine, (Control +ve)

Group (2): Sheep vaccinated with inactivated oil trivalent FMD vaccine containing (Vit. A)

Group (3): Sheep vaccinated with inactivated oil trivalent FMD vaccine containing (Vit. E-selenium)

Group (4): Sheep vaccinated with inactivated oil trivalent FMD vaccine containing (Vit. A \& E-selenium)

- Serum neutralization antibody titer was expressed as $\log _{10}$

- $\quad$ Protective serum antibody titer $=1.5 \log _{10}$ according to OIE (2012)

* There is a significant increase between Group (4) and Group (3), also between Group (3) and Group (2) (at $\mathrm{P}<0.05$ )

** There is a significant increase between Group (4) and Group (1), also between Group (4) and Group (2) (at $\mathrm{P}<0.01$ )

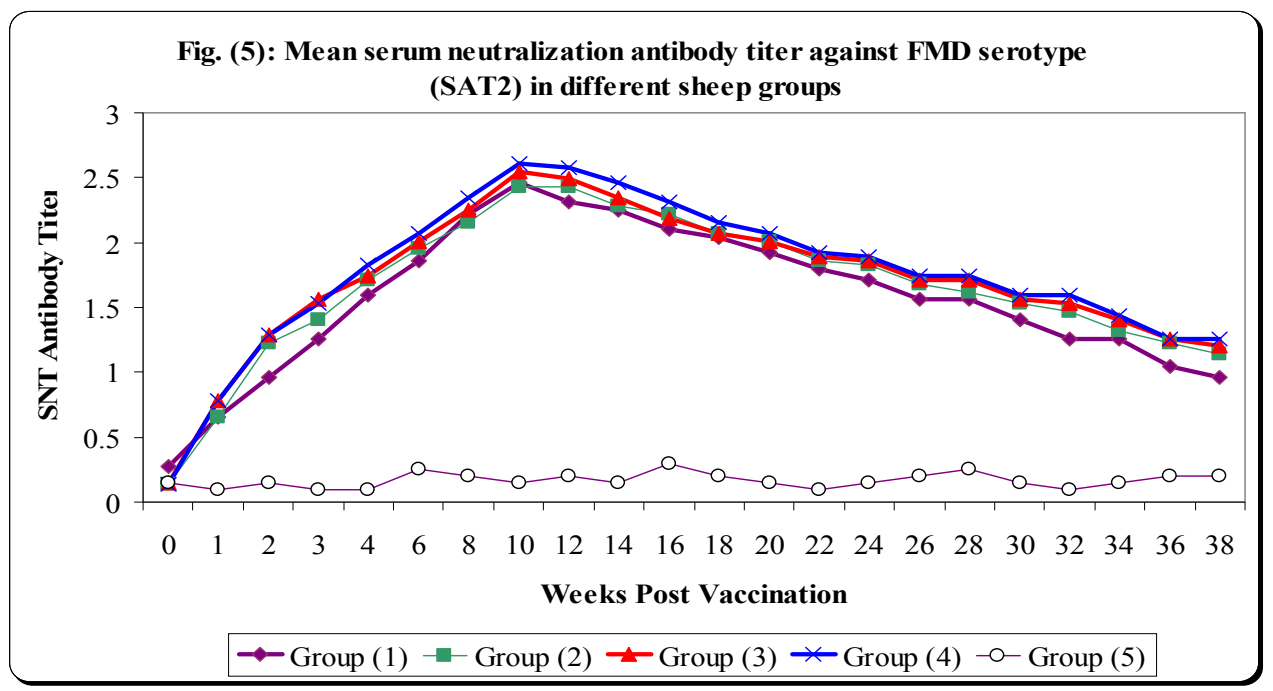


Table 6. Mean ELISA antibody titer against FMD serotype (SAT2) in different sheep groups

\begin{tabular}{|c|c|c|c|c|c|}
\hline \multirow{2}{*}{ Weeks Post } & \multicolumn{5}{|c|}{ Sheep Groups } \\
\cline { 2 - 6 } & Group (1) & Group (2) & Group (3)* & Group (4)** & $\begin{array}{c}\text { Control -ve } \\
\text { sheep group }\end{array}$ \\
\hline 0 & 0.20 & 0.14 & 0.15 & 0.15 & 0.30 \\
\hline 1 & 0.96 & 0.93 & 0.93 & 0.96 & 0.25 \\
\hline 2 & 1.26 & 1.50 & 1.59 & 1.53 & 0.30 \\
\hline 3 & 1.68 & 1.80 & 1.80 & 1.74 & 0.30 \\
\hline 4 & 1.89 & 2.01 & 1.95 & 1.98 & 0.25 \\
\hline 6 & 1.95 & 2.04 & 2.10 & 2.07 & 0.20 \\
\hline 8 & 2.25 & 2.34 & 2.37 & 2.49 & 0.20 \\
\hline 10 & 2.46 & 2.52 & 2.55 & 2.64 & 0.20 \\
\hline 12 & 2.43 & 2.55 & 2.55 & 2.64 & 0.25 \\
\hline 14 & 2.25 & 2.49 & 2.40 & 2.49 & 0.30 \\
\hline 16 & 2.10 & 2.31 & 2.34 & 2.40 & 0.30 \\
\hline 18 & 2.04 & 2.13 & 2.28 & 2.25 & 0.30 \\
\hline 20 & 1.92 & 1.98 & 2.16 & 2.07 & 0.30 \\
\hline 22 & 1.86 & 1.89 & 2.04 & 1.92 & 0.25 \\
\hline 24 & 1.77 & 1.83 & 2.01 & 1.89 & 0.20 \\
\hline 26 & 1.65 & 1.74 & 1.86 & 1.74 & 0.20 \\
\hline 28 & 1.59 & 1.68 & 1.89 & 1.59 & 0.20 \\
\hline 30 & 1.50 & 1.56 & 1.71 & 1.59 & 0.20 \\
\hline 32 & 1.35 & 1.41 & 1.53 & 1.59 & 0.25 \\
\hline 34 & 1.23 & 1.26 & 1.56 & 1.47 & 0.20 \\
\hline 36 & 1.26 & 1.20 & 1.35 & 1.41 & 0.20 \\
\hline 38 & 1.08 & 1.02 & 1.35 & 1.32 & 0.20 \\
\hline
\end{tabular}

Group (1): Sheep vaccinated with inactivated oil trivalent FMD vaccine, (Control +ve)

Group (2): Sheep vaccinated with inactivated oil trivalent FMD vaccine containing (Vit. A)

Group (3): Sheep vaccinated with inactivated oil trivalent FMD vaccine containing (Vit. E-selenium)

Group (4): Sheep vaccinated with inactivated oil trivalent FMD vaccine containing (Vit. A \& E-selenium)

- $\quad$ ELISA antibody titer was expressed as $\log _{10}$

- $\quad$ Protective serum antibody titer $=1.65 \log _{10}$ according to OIE (2012)

* There is a significant increase between Group (4) and Group (3), also between Group (3) and Group (2) (at $\mathrm{P}<0.05$ )

** There is a significant increase between Group (4) and Group (1), also between Group (4) and Group (2) (at $\mathrm{P}<0.01$ )

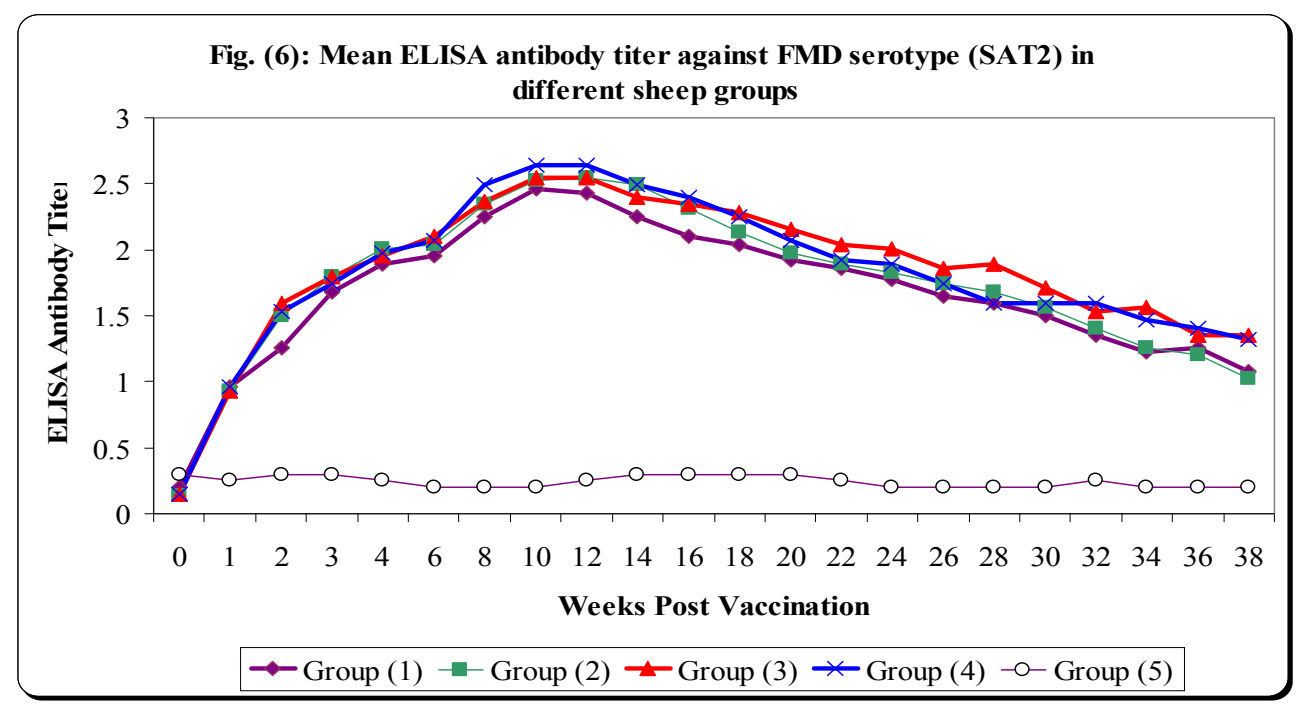


Table 7. Cell mediated immune response (expressed as Delta Optical Density) of vaccinated sheep were vaccinated with different inactivated trivalent FMD vaccines

\begin{tabular}{|c|c|c|c|c|c|c|c|c|c|}
\hline & \multirow{2}{*}{ Groups } & \multicolumn{8}{|c|}{ Days Post Vaccination } \\
\hline & & 0 & 3 & 7 & 14 & 21 & 28 & 35 & 42 \\
\hline \multirow{2}{*}{1} & Non-specific mitogen & 0.051 & 0.121 & 0.324 & 0.383 & 0.510 & 0.463 & 0.340 & 0.265 \\
\hline & FMDV as specific mitogen & 0.060 & 0.231 & 0.420 & 0.490 & 0.600 & 0.550 & 0.490 & 0.350 \\
\hline \multirow{2}{*}{2} & Non-specific mitogen & 0.067 & 0.197 & 0.401 & 0.464 & 0.600 & 0.565 & 0.480 & 0.390 \\
\hline & FMDV as specific mitogen & 0.070 & 0.289 & 0.500 & 0.602 & 0.710 & 0.671 & 0.620 & 0.495 \\
\hline \multirow{2}{*}{$3 *$} & Non-specific mitogen & 0.055 & 0.245 & 0.490 & 0.522 & 0.711 & 0.650 & 0.557 & 0.415 \\
\hline & FMDV as specific mitogen & 0.076 & 0.345 & 0.589 & 0.700 & 0.803 & 0.755 & 0.700 & 0.600 \\
\hline \multirow{2}{*}{$4 * *$} & Non-specific mitogen & 0.059 & 0.271 & 0.574 & 0.646 & 0.820 & 0.768 & 0.691 & 0.470 \\
\hline & FMDV as specific mitogen & 0.065 & 0.400 & 0.610 & 0.790 & 0.910 & 0.835 & 0.800 & 0.630 \\
\hline \multirow{2}{*}{5} & Non-specific mitogen & 0.053 & 0.064 & 0.057 & 0.051 & 0.060 & 0.058 & 0.067 & 0.069 \\
\hline & FMDV as specific mitogen & 0.061 & 0.059 & 0.053 & 0.060 & 0.054 & 0.052 & 0.061 & 0.060 \\
\hline
\end{tabular}

Group (1): (Control positive group) Sheep vaccinated with inactivated trivalent FMD vaccine

Group (2): Sheep vaccinated with inactivated trivalent FMD vaccine containing Vit. A.

Group (3): Sheep vaccinated with inactivated trivalent FMD vaccine containing Vit. (E-selenium).

Group (4): Sheep vaccinated with inactivated trivalent FMD vaccine containing both Vit. A and Vit. (E-selenium).

Group (5): (Control negative group) sheep non-vaccinated

* There is a significant increase between Group (4) and Group (3), also between Group (3) and Group (2) (at P < 0.05$)$

** There is a significant increase between Group (4) and Group (1), also between Group (4) and Group (2) (at P < 0.01) 


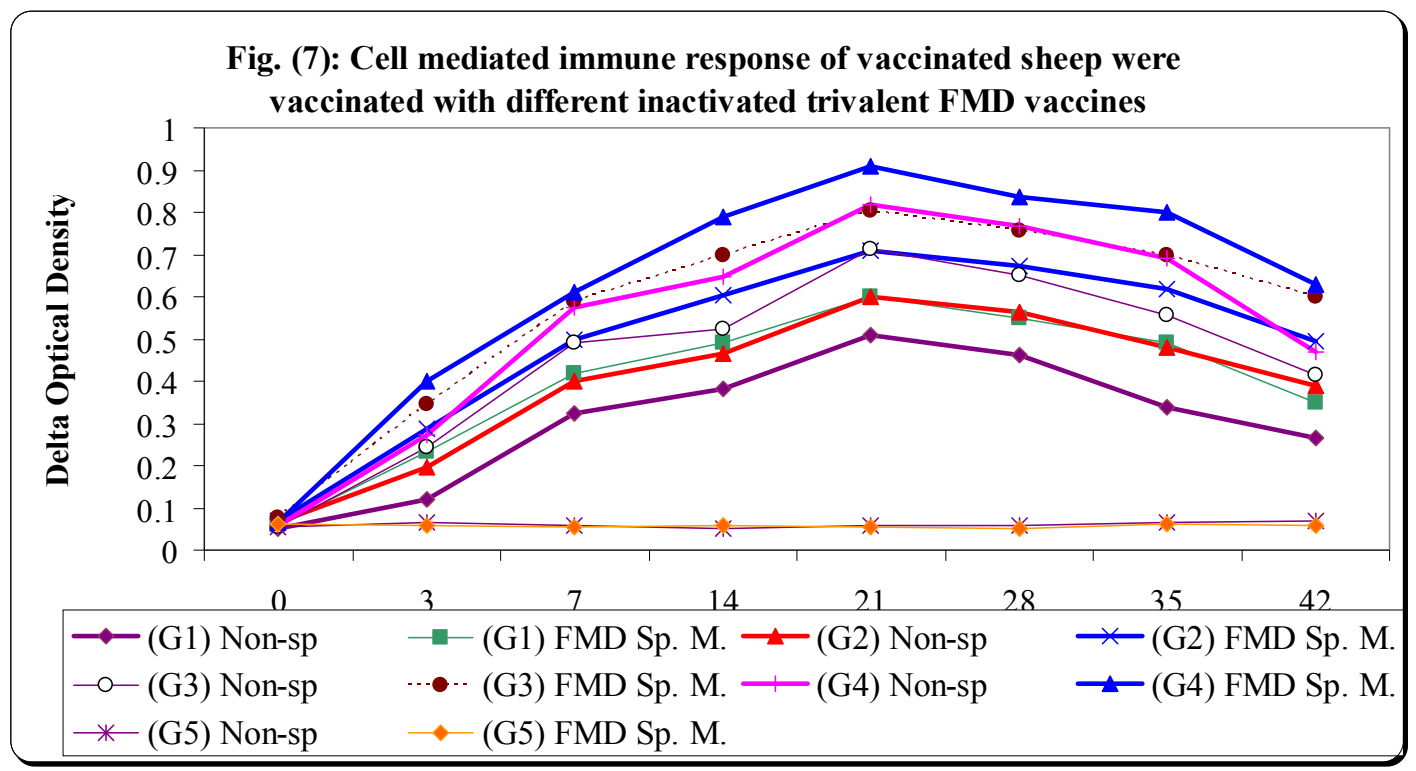

\section{REFERENCES}

1. Abd El-Rahman, A. O.; Farag, M. A.; Samira El- Kilany, Ali ,S. M. and Manal Abo Yazed 2006. Isolation and identification of Serotype $O$ of Foot and Mouth Disease virus from imported bulls and its correlation to the current used vaccine strain O1/3/1993. Proc. $3^{\text {rd }}$ Inter Conf. Vet. Res. Div., NRC, Cairo, Egypt, pp. 91-100.

2. Abeer, E.M.; Fatma, A.A.; Eman, M. and Abo Bakr, A. 2009. Study of humoral and cellular immune response in FMD vaccinated sheep. Egypt. Vet. Med., 69: 101108.

3. Aidaros, H.A. 2002. Regional status and approaches to control and eradication of foot and mouth disease. In the Middle and North Africa. Rev. Sci. Tech. Off. Int. Epiz., 21: 451-458.

4. Bahl, R., N. Bhandari, S. Kant, K. Molbak, E. Ostergaard, and M. K. Bhan. 2002. Effect of vitamin A administered at Expanded Program on Immunization contacts on antibody response to oral polio vaccine. Eur. J. Clin. Nutr. 56:321-325.

5. Barteling, S.J. and Vreeswij, K.J. 1991. "Development in foot and mouth disease vaccine". Vaccine, 9(2): 75-88.

6. Cherly, F. Nockels 1996. Antioxidants improve cattle immunity following stress. Animal Feed Science Technology, 62: 59-68.

7. Clo, M.; Dungu, B.; Vanstanden, L.; Cassim, N. and Vosloo, N. 2008. Evaluation of different adjuvants for Foot and Mouth Disease vaccine containing all the SAT serotypes.Onderstepoort Journal of Veterinary Research 75: 17-31.

8. Coutsoudis, A., Kiepiela, P., Covadia, H. and Boughton, M. 1992. Vitamin A supplementation enhances specific IgG antibody levels and total lymphocyte 
numbers while improving morbidity in measles. Pediatr. Infect. Dis. J., 11:200209.

9. Farag, M.A., Aggour, M.A. and Daoud, A.M. 2005. ELISA as a rapid method for detecting the correlation between the field isolates of Foot and Mouth Disease and the current used vaccine strain in Egypt. Vet. Med. J., Giza. Vol. 53, No. 4: 949955.

10. Hamblin, C., Barnett, I. T. R. and Hedger, R. S. 1986. A new enzyme-linked immunosorbent assay (ELISA) for the detection of antibodies against foot-andmouth disease virus. I. Development and method of ELISA. Journal of Immunological Methods 93, 115-121.

11. Heba Atia 2017. Comparative studies on trivalent FMD inactivated vaccine adjuvanted with Montanide ISA-206 and ISA-201. Ph.D. Thesis, Virology, Fac. Vet Med., Benha University.

12. Hogan, J.S.; Smith, K.L.; Weiss, W.P.; Tod, D.A. Hunter and Schockey, W.L. 1990. Relation among vitamin $\mathrm{E}$, selenium and bovine blood neutrophils. J. Dairy Sci., 73: 2372-2378.

13. Howard, T. Petro; Lynell W. Klassen and David, Kay 1989. Selenium and the immune response. 1 . Modulation of Allo reactive human lymphocyte function invitro. J. Leuko, 45: 207-217.

14. Hunter, P. 1998. Vaccination as a mean of control of FMD in sub-Saharan Africa. Vaccine 16 (2-3): 261-264.

15. Jason, J., Archibald L.K., Nwanyanwu O.C., Sowell A.L., Buchanan I., Larned J., Bell M., Kazembe P.N., Dobbie H., and Jarvis W.R. 2002. Vitamin A levels and immunity in humans. Clin. Diagn. Lab. Immunol. 9:616-621.

16. Kanchana, G. and Jeyanthi, G.P. 2010. Influence of vitamin E and selenium supplement on the growth performance and antibody responses of layer. Int. J. Pharma. Biosci., V1 (2): 1-9.

17. Kutukculer, N.T., Akil, A. Eggmeen, Kutugobs Axsit Z., Ozmen D., Turgan N., Bayindir O.and Caglayam S. 2000. Adequate immune response to tetanus toxoid and failure of vitamin $A$ and $E$ supplementation to enhance antibody response in healthy children. Vaccine, 18: 2979-2984.

18. Lockart, C.; Sumption, K.; Pino, J. and Lubroth, J. 2012. Foot and mouth disease caused by serotype SAT2 in Egypt and Libya. Empress Watch, 25: 1-7.

19. Martin Nemec; Hidiroglou, M.; Nielsen, K. and Prouix, J. 1990. Effect of vitamin E and selenium supplementation on some immune parameters following vaccination against brucellosis in cattle. J. Anim. Sci., 68: 4303-4309. 
20. Milad, K., Racz, O, Sipulova, A., Bajova, V. and Kovac, G. 2001. Effect of vitamin E and selenium on blood glutathione peroxidase activity and serum immunological parameters in sheep. Vet. Med. Gzen, 46 (1): 1-5.

21. OIE. 2012. Foot and Mouth Disease. Chapter 2.1.5. In Manual of Standard for Diagnostic Test and Vaccine. Paris, pp. 77-92.

22. Patil, P.K., Bayry, J., Ramakrishna, C., Hugar, B., Misra, L.D. and Natarajan, C. 2002. Immune response of goats against foot and mouth disease quadrivalent vaccine: comparison of double oil emulsion and aluminum hydroxide gel vaccine in eliciting immunity. Vaccine, 20:2781-2789.

23. Pereira, H.G. 1977. Subtyping of FMD. Int. Symp. FMD, Lyon Develop. Biol. Standard, 35: 167-174.

24. Rahman, M. M., Mahalanabis D., Hossain S., Wahed M. A., Alvarez J. O., Siber G. R., Thompson C., Santosham M., and Fuchs G. J. 1999. Simultaneous vitamin A administration at routine immunization contact enhances antibody response to diphtheria vaccine in infants younger than six months. J. Nutr. 129:2192-2195.

25. Rajeesh, M., Dass, R.S., Garg, A.K. and Chaturvedi, V.K. 2008. Effect of vitamin E supplementation on serum alpha tocopherol and immune status of Murrah buffalo (Bubalus bubalis) calves. J. Anim. Feed Sci., 17: 19-29.

26. Reffett Stabel, J.W.; Spears, T.T.; Brown, Jr and Brake, J. 1989. Selenium effect on glutathione peroxidase and immune response of stressed calves challenged with Pasteurella Hemolytica. J. Anim. Sci., 67 (7): 557-564.

27. Ribeiro, O. G., Maria D. A., Adriouch S., Pechberty S., Cabrera W. H., Morisset J., Ibanez O. M., and Seman M. 2003. Convergent alteration of granulopoiesis, chemotactic activity, and neutrophil apoptosis during mouse selection for high acute inflammatory response. J. Leukoc. Biol. 74:497-506.

28. Sendecor, G.W. 1971. Statistical Methods, $4^{\text {th }}$ Ed. Iowa State Univ. Press.

29. Semba, R. D. 1999. Vitamin A and immunity to viral, bacterial and protozoan infections. Proc. Nutr. Soc. 58:719-727.

30. Semba, R. D., Muhilal, Scott A. L., Natadisastra G., Wirasasmita S., Mele L., Ridwan E., West, Jr. K. P., and Sommer A. 1992. Depressed immune response to tetanus in children with vitamin A deficiency. J. Nutr. 122:101-107.

31. Semba, R. D., Lyles C.M., Margolick J.B., Caiaffa W.T., Farzadegan H., Cohn, S.and Vlahov D. 1998. Vitamin A supplementation and human immunodeficiency virus load in injection drug users. J. Infect. Dis. 177:611- 616.

32. Semba, R. D., C. Ndugwa, R. T. Perry, T. D. Clark, J. B. Jackson, G. Melikian, J. Tielsch, and F. Mmiro 2005. Effect of periodic vitamin A supplementation on 
mortality and morbidity of human immunodeficiency virus-infected children in Uganda: a controlled clinical trial. Nutrition 21: 25-31.

33. Sharma, R. and Kakkar, N.K. 2005. Scenario of Foot and Mouth Disease outbreaks in Harana State during the years 2003 and 2004. Haryana Vet., 44: 42-45.

34. Sharma, M.C.; Pathak, N.N.; Huag, N.N.; Nhi, D.L. and Vue, N.V. 1985. Report on the outbreaks of Foot and Mouth Disease in Morrah buffloes reared in Southern part of Vietnam. A.J. Della Porta, Sydney, Australia, ACD Press, 302-303.

35. Sonia, A.M. 2007. Studies on the preparation of an improved Foot and Mouth Disease oil vaccine. Ph. D. Thesis of Virology, Faculty of Veterinary Medicine, University of Cairo.

36. Soos, T. and Tuboly, S. 1983. Study of immunogenicity of three aphthovirus strain. 1. Cellular immunity. Mag. Allatrov. Lapia, 38: 341-344.

37. Stephensen, C. B. 2001. Vitamin A, infection, and immune function. Annu. Rev. Nutr. 21:167-192.

38. Tengerdy, R.P. 1990. The role of vitamin E in immune response and disease resistance. Ann. N.Y. Acad. Sci. Press.

39. Verma A.K. 2008. Studies on molecular epidemiology of FMD in bovines with special reference to serological diagnosis of vaccinated and infected animals. PhD. Thesis, Pandit Deen Dayal Ypadhyaya Pashu-Chikitsa Vigyan Vishwavidyalaya Evan Go Anusend hen Thura, India.

40. Verma A.K. 2010. Evaluation of the MTT Iymphocyte proliferation assay for the diagnosis of neurocystic ercosis. J. Microbiol. Methods, 81: 175-178.

41. Villamor, E. and Fawzi, W. 2005. Effect of vitamin A supplementation on immune responses and correlation with clinical outcomes. Clin. Micro., 18 (3): 446-464.

42. Weber, M.; Fodor, J.; Balogh, K.; Wagner, L.; Erdelyi, M. and Hezes, M. 2008. Effect of vitamin supplementation on immunity against Newcastle virus in T2-toxin challenged chicks. Acta Vet. Brno, 11: 45-49.

43. Wuryastuti, H.D.; Stowe, R.W.; Bull, R.W. and Miller, E.R. 1993. Effect of vitamin $E$ and selenium on immune response of peripheral blood, colostrum and milk leukocytes of sows. J. Anim. Sci., 71: 2464-2472.

44. Zahran, G.E.D. 1960. Foot and Mouth Disease in Southern region of URA. Bull. Off. Int. Epiz., 15: 390-393. 


\title{
دراسة المناعة المصلية والخلوية فى الأغنام المحصنة بأنواع مختلفة من لقاحات الحمى القلاعية المثبطة
}

\author{
"عبير حسن محمد حسن'، ياقوت عبد الفتاح السنوسى'، \\ عفاف دسوقى عبد المجيد، ابو بكر احمد عجور'، عبير عزت محمود' \\ 1 ـ قسم الحمى القلاعية - معهد الامصال و اللقاحات البيطرية - العباسية - القاهرة \\ r ا ـ الكبياء الحبوية - كلية الطب البيطرى - جامعة بنها
}

صمدت هذه الدر اسة كمحاولة لدقارنة تأثثر كلاً من لقاح الحمى القلاعية المنتج حالباً ولقاح

الحمى القلاعية مضاف اليه فيتامين "A" ولقاح الحمى القلاعية مضاف إليه فيتامين "E" سيلينيوم ونفس اللقاح مضاف إليه كلاً من فيتامين "E" - "Eلينيوم معاً. وقد أوضحت النتائج أن المناعة المصلية

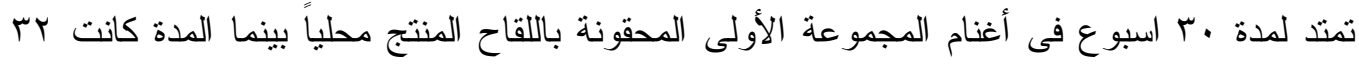

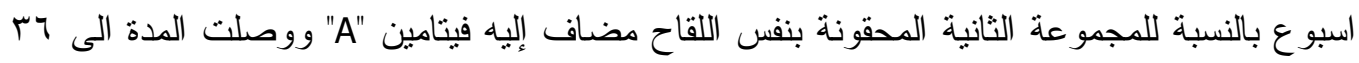

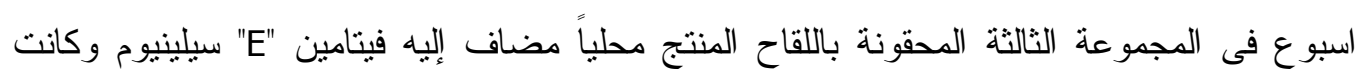

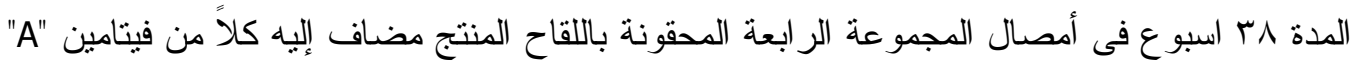

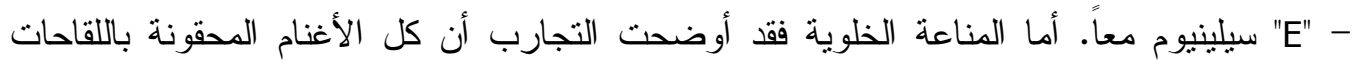
المختلفة للحمى القلاعية أعطت أعلى منوسط حسابى للكثافة الضوئية (Optical Density) بعد الحقن ب اب بوم وذللك باستخدام تقنية (MTT-Kit) و التى تقيس الميتوجين غير المتخصصة للحمى القلاعية

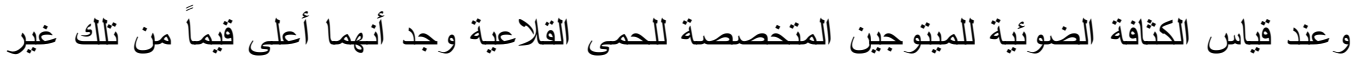
المتخصصة وذلك فى الأغنام المحصنة بلقاح الحمى القلاعية مضاف إليه فيتامين - "E" - سيلينيوم 